\title{
UNITARY PARTS OF GENERALIZED TOEPLITZ OPERATORS
}

\author{
JEFFREY R. BUTZ
}

\begin{abstract}
For a generalized Toeplitz operator acting on a Hilbert space with unilateral shift, necessary and sufficient conditions are obtained for the existence of a nontrivial unitary part, and an explicit description of this unitary part is given.
\end{abstract}

Let $T$ be a contractive linear operator on a separable complex Hilbert space $X$. Such an operator is said to be completely nonunitary if it has no nontrivial reducing subspace $N$ such that the restriction $T \mid N$ of $T$ to $N$ is unitary. It is known [2, Theorem I.3.2] that for any contraction $T$ on $X$ we can find a unique orthogonal decomposition $X=M \oplus M_{1}$ such that $M$ and $M_{1}$ reduce $T, T \mid M$ is unitary and $T \mid M_{1}$ is completely nonunitary. It is not excluded that $M$ or $M_{1}$ is possibly the subspace $\{0\}$. Furthermore, $M$ is given by $M=\left\{x \in X:\left\|T^{n} x\right\|=\|x\|=\left\|T^{* n} x\right\|, n=1,2, \ldots\right\}$ and is called the unitary subspace of $T . T \mid M$ is called the unitary part of $T$.

In this paper, we describe explicitly the above decomposition for the class of generalized Toeplitz operators, which are obtained as follows.

We say that an isometry $S: X \rightarrow X$ is a unilateral shift if there exists a subspace $C$ in $X$ for which $\left(S^{j} C\right) \perp\left(S^{k} C\right)$ for nonnegative integers $j \neq k$, and

$$
X=\bigoplus_{n=0}^{\infty} S^{n} C \text {. }
$$

It is not difficult to see that $C$ is uniquely determined by $S$, viz., $C=(S X)^{\perp}$.

In what follows, we shall find it convenient to consider the minimal unitary extension $U$ of $S$. Then $U$ is a bilateral shift acting on a Hilbert space $Y$ containing $X$, and $Y$ decomposes into the orthogonal direct sum

$$
Y=\bigoplus_{n=-\infty}^{\infty} U^{n} C \text { and } U \mid X=S .
$$

That such a space $Y$ exists is clear, since it can be constructed as a direct sum of a countable number of copies of $C$ indexed on the integers. Equivalently, $U$ and $Y$ are obtained as the minimal unitary dilation of $S$ in the structure theory of Sz.-Nagy and Foias [2, Chapter I].

We now fix a unilateral shift $S$ on the Hilbert space $X$ and make the following definitions.

Definitions. 1. A bounded operator $T: X \rightarrow X$ is Toeplitz if $S^{*} T S=T$.

Received by the editors May 24, 1976 and, in revised form, February 7, 1977.

AMS (MOS) subject classifications (1970). Primary 47A20, 47B35.

(1) American Mathematical Society 1977 
2. A bounded operator $L: Y \rightarrow Y$ is Laurent if $L U=U L$.

These operators have been previously studied by Rosenblum and Page, cf. [4], and they are generalizations of the classical Toeplitz and Laurent operators, where $X$ is the Hardy space $H^{2}$ consisting of those $L^{2}$ functions defined on the unit circle whose Fourier coefficients vanish on the negative integers and $S$ is equal to multiplication by $z$. In particular, we have the following fact which, in this generalized setting, has been observed by Page [3].

Proposition (PAge [3]). A bounded operator $T$ on $X$ is Toeplitz if and only if there exists a bounded Laurent operator $L$ on $Y$ such that $T=P_{+} L \mid X$, where $P_{+}: Y \rightarrow X$ is the orthogonal projection. In this case, $\|L\|=\|T\|$.

This generalizes the fact that, in the classical case, Toeplitz operators are of the form $T_{\varphi}: x\left(e^{i \theta}\right) \mapsto P_{+} \varphi\left(e^{i \theta}\right) x\left(e^{i \theta}\right)$, where $\varphi \in L^{\infty}$ and $P_{+}: L^{2} \rightarrow H^{2}$ is the orthogonal projection. In such a case, we have $\left\|T_{\varphi}\right\|=\|\varphi\|_{\infty}$ and $T_{\varphi}^{*}=$ $T_{\bar{\phi}}$. For these classical Toeplitz operators, Goor [1] has proved the following.

THEOREM (GoOR [1]). If $\varphi \in L^{\infty},\|\varphi\|_{\infty} \leqslant 1$, and $\varphi$ is (almost everywhere) nonconstant, then $T_{\varphi}$ is completely nonunitary.

We now obtain a theorem which generalizes to abstract Toeplitz operators this characterization of unitary parts. Unlike classical Toeplitz operators, however, nontrivial abstract Toeplitz operators can have nontrivial unitary parts. A simple example of this is given by $X=H^{2} \oplus H^{2}$ with $T: X \rightarrow X$ defined by $T=T_{0} \oplus T_{1}$, where $T_{0}: H^{2} \rightarrow H^{2}$ is multiplication by a constant of modulus 1 , and $T_{1}: H^{2} \rightarrow H^{2}$ is a completely nonunitary Toeplitz operator. Then $T$ has the nontrivial unitary subspace $M=H^{2} \oplus\{0\}$, and the restriction of $T$ to $M$ is multiplication by a constant of modulus 1 (which is a unitary operator on the 1-dimensional generating subspace for $M$ ). Our theorem shows that all abstract Toeplitz contractions decompose in a similar way.

TheOREM. Let $T: X \rightarrow X$ be a Toeplitz contraction on the Hilbert space $X=\bigoplus_{n=0}^{\infty} S^{n} C$. Then there exists a decomposition $C=C_{0} \oplus C_{1}$ such that $M=\bigoplus_{n=0}^{\infty} S^{n} C_{0}, M^{\perp}=\bigoplus_{n=0}^{\infty} S^{n} C_{1}$, where $M$ is the unitary subspace of $T$, and $x=\sum_{n=0}^{\infty} S^{n} c_{n} \in M \Leftrightarrow c_{n} \in C_{0}$ for $n=0,1,2, \ldots$ Furthermore, if $M \neq$ $\{0\}$ (i.e., $C_{0} \neq\{0\}$ ), then there exists a unitary operator $R_{0}: C_{0} \rightarrow C_{0}$ such that for $x=\sum_{n=0}^{\infty} S^{n} c_{n} \in M$ we have $T x=\sum_{n=0}^{\infty} S^{n} R_{0} c_{n}$.

Proof. By the above proposition, there exists an operator $L: Y \rightarrow Y$ such that $P_{+} L x=T x$ for $x \in X,\|L\|=\|T\| \leqslant 1$, and $U L=L U$. Suppose that there exists a nonzero $x$ in the unitary subspace $M$ of $T$, which, as indicated above, is given by $M=\left\{x \in X:\left\|T^{n} x\right\|=\|x\|=\left\|T^{* n} x\right\|, n=1,2, \ldots\right\}$. Then, in particular, 


$$
\|x\|=\|T x\|=\left\|P_{+} L x\right\| \leqslant\|L x\| \leqslant\|x\|,
$$

so that $L x=T x \in X$. Similarly, $L^{*} x=T^{*} x \in X$. Continuing for $n=$ $2,3, \ldots$, we obtain

$$
\begin{array}{r}
M=\left\{x \in X: L^{n} x, L^{* n} x \in X \text { and }\left\|L^{n} x\right\|=\|x\|=\left\|L^{* n} x\right\|,\right. \\
n=1,2, \ldots\} .
\end{array}
$$

Also, for $x \in M$ we have $L^{n} S x=S L^{n} x \in X$ and $\left\|L^{n} S x\right\|=\left\|S L^{n} x\right\|=\|x\|$ $=\|S x\|$, and similarly for $L^{* n}$. Therefore, the subspace $M$ is invariant under the unilateral shift. Then the restriction of $S$ to $M$ is a shift since

$$
\bigcap_{k=0}^{\infty} S^{k} M \subseteq \bigcap_{k=0}^{\infty} S^{k} X=\{0\},
$$

and thus we can write $M=\bigoplus_{n=0}^{\infty} S^{n} C_{0}$, where $C_{0}=(S M)^{\perp} \cap M$. We claim that the subspace $C_{0}$ is reducing for $L$ (and hence $T$ ). Let $c \in C_{0}$, then by (1) $c \in M \Rightarrow L c \in M$, and for $x \in M$ we have $\langle L c, S x\rangle=\left\langle c, L^{*} S x\right\rangle=$ $\left\langle c, S L^{*} x\right\rangle=0$, since $S L^{*} x \in S M$ and $c \in(S M)^{\perp}$. Therefore, $L c \in M$ and similarly $L^{*} c \in M$, so that $C_{0}$ reduces $L$ as claimed.

Writing now $x=\sum_{n=0}^{\infty} S^{n} c_{n} \in M, c_{n} \in C_{0}$, we obtain

$$
T x=L x=L\left(\sum_{n=0}^{\infty} S^{n} c_{n}\right)=\sum_{n=0}^{\infty} S^{n} L c_{n}=\sum_{n=0}^{\infty} S^{n} R_{0} c_{n},
$$

where $R_{0}=L \mid C_{0}$. Also, for $S^{*} x=\sum_{n=0}^{\infty} S^{n} c_{n+1}$ we have

$$
L S^{*} x=L\left(\sum_{n=0}^{\infty} S^{n} c_{n+1}\right)=\sum_{n=0}^{\infty} S^{n} L c_{n+1}=\sum_{n=0}^{\infty} S^{n} R_{0} c_{n+1} \in X,
$$

so that $\left\|T S^{*} x\right\|=\left\|L S^{*} x\right\|=\left\|S^{*} x\right\|$, and similarly for $T^{*}$ and powers $n=$ $2,3, \ldots$ Hence, $M$ is in fact a reducing subspace for the shift.

We now claim that $C_{0}=C \cap M$. It is clear that $C \cap M=(S X)^{\perp} \cap M$ $\subseteq(S M)^{\perp} \cap M=C_{0}$, so we must show that if $x \in(S M)^{\perp} \cap M$, then $x \in$ $(S X)^{\perp} \cap M$. For $y \in X$ write $y=y_{1}+y_{2}$ with $y_{1} \in M, y_{2} \in M^{\perp}$, then

$$
\langle x, S y\rangle=\left\langle x, S y_{1}\right\rangle+\left\langle x, S y_{2}\right\rangle=0+\left\langle S^{*} x, y_{2}\right\rangle=0,
$$

so that $x \in(S X)^{\perp} \cap M$ as required.

It remains to show that for $C_{1}=C \ominus C_{0}$ we have $M^{\perp}=\bigoplus_{n=0}^{\infty} S^{n} C_{1}$.If $\sum_{n=0}^{\infty} S^{n} c_{n}^{\prime} \in \bigoplus_{n=0}^{\infty} S^{n} C_{1}$ and $\sum_{m=0}^{\infty} S^{m} c_{m} \in M, c_{n}^{\prime} \in C_{1}, c_{m} \in C_{0}$, then

$$
\left\langle\sum_{n=0}^{\infty} S^{n} c_{n}^{\prime}, \sum_{m=0}^{\infty} S^{m} c_{m}\right\rangle=\sum_{m=0}^{\infty}\left\langle S^{n} c_{n}^{\prime}, S^{n} c_{n}\right\rangle=\sum_{n=0}^{\infty}\left\langle c_{n}^{\prime}, c_{n}\right\rangle=0
$$

so that $\bigoplus_{n=0}^{\infty} S^{n} C_{1} \subseteq M^{\perp}$. Conversely, suppose that we had $x=\sum_{n=0}^{\infty} S^{n} c_{n} \in$ $M^{\perp}$ with $c_{k} \notin C_{1}$ for some integer $k$. Then write $c_{k}=c_{k}^{\prime}+c_{k}^{\prime \prime}$ with $c_{k}^{\prime} \in C_{0}$, $c_{k}^{\prime \prime} \in C_{1}, c_{k}^{\prime} \neq 0$. Since $S^{k} c_{k}^{\prime} \in M$, we would then get 


$$
\left\langle x, S^{k} c_{k}^{\prime}\right\rangle=\left\langle\sum_{n=0}^{\infty} S^{n} c_{n}, S^{k} c_{k}^{\prime}\right\rangle=\left\langle S^{k} c_{k}, S^{k} c_{k}^{\prime}\right\rangle=\left\langle c_{k}^{\prime}, c_{k}^{\prime}\right\rangle \neq 0,
$$

which contradicts that $x \in M^{\perp}$. Therefore, $M^{\perp}=\bigoplus_{n=0}^{\infty} S^{n} C_{1}$, and the proof is complete.

REMARKS. 1. As a converse to the above theorem, note that any orthogonal decomposition $C=C_{0} \oplus C_{1}$ together with a unitary operator $R_{0}: C_{0} \rightarrow$ $C_{0}$ determines a Toeplitz operator $T$ whose unitary subspace is given by $M=\bigoplus_{n=0}^{\infty} S^{n} C_{0}$. For example, define $T$ by

$$
T\left(\sum_{n=0}^{\infty} S^{n} c_{n}\right)=T\left(\sum_{n=0}^{\infty} S^{n}\left(c_{n}^{\prime}+c_{n}^{\prime \prime}\right)\right)=\sum_{n=0}^{\infty} S^{n} R_{0} c_{n}^{\prime},
$$

where $c_{n}=c_{n}^{\prime}+c_{n}^{\prime \prime} \in C, c_{n}^{\prime} \in C_{0}, c_{n}^{\prime \prime} \in C_{1}$.

2. The special case of $\operatorname{dim} C=1$, as described by Goor, follows as an easy consequence of the theorem. Either $\operatorname{dim} C_{0}=0$, so that $M=\{0\}$ and $T$ is completely nonunitary, or $\operatorname{dim} C_{0}=1$ and $X=\bigoplus_{n=0}^{\infty} S^{n} C_{0}=M$. In the latter case, for $x \in X, x=\sum_{n=0}^{\infty} S^{n} c_{n}$ for some $c_{n} \in C_{0}$, we have by the theorem that $T x=\sum_{n=0}^{\infty} S^{n} R_{0} c_{n}$, where $R_{0}$ is a unitary operator on $C_{0}$. But then $R_{0}$ must be multiplication by a scalar $\alpha$ of modulus 1 , which implies that $T$ is itself multiplication by the scalar $\alpha$, as asserted in Goor's result.

ACKnowledgment. The author wishes to thank Professor Philip Hartman for his encouragement and many helpful suggestions.

\section{REFERENCES}

1. R. Goor, On Toeplitz operators which are contractions, Proc. Amer. Math. Soc. 34 (1972), 191-192.

2. B. Sz.-Nagy and C. Foias, Harmonic analysis of operators on Hilbert space, North-Holland, Amsterdam, 1970.

3. L. Page, Bounded and compact vectorial Hankel operators, Trans. Amer. Math. Soc. 150 (1970), 529-540.

4. M. Rosenblum, Self-adjoint Toeplitz operators, Summer Institute of Spectral Theory and Statistical Mechanics 1965, Brookhaven National Laboratory, Upton, New York, 1966.

Department of Mathematics, University of OkLahoma, Norman, Oklahoma 73019 